\title{
LETTER
}

\section{Diagnosis and treatment of COVID-19 associated pulmonary apergillosis in critically ill patients: results from a European confederation of medical mycology registry}

\author{
Juergen Prattes ${ }^{1}(\mathbb{0})$, Joost Wauters ${ }^{2^{*}}\left(\mathbb{D}\right.$, Daniele Roberto Giacobbe ${ }^{3,4}\left(\mathbb{D}\right.$, Katrien Lagrou ${ }^{5,6}$ (1) \\ and Martin Hoenigl 1,7,8* on behalf of the ECMM-CAPA Study Group
}

(c) 2021 Springer-Verlag GmbH Germany, part of Springer Nature

Dear Editor,

Coronavirus disease 2019 (COVID-19)-associated pulmonary aspergillosis (CAPA) has emerged as an important complication among patients with acute respiratory failure caused by SARS-CoV-2 [1-3]. A cornerstone in CAPA diagnosis is microbiology, including culture from lower respiratory tract samples, and bronchoalveolar lavage fluid (BALF) galactomannan (GM) $[4,5]$.

We performed a multinational cohort study including 20 centers in 9 countries with the aim to evaluate diagnostic approaches and treatment of CAPA as well as CAPA prevalence. All participating centers were invited to provide data on diagnostic work-up, treatment and outcome on prospective cohorts of consecutive COVID19 ICU patients (inclusion criteria: SARS-CoV-2-positive polymerase chain reaction, admission to intensive care unit (ICU) due to respiratory failure) with and without CAPA via an online case report form between 03/2020 and $05 / 2021$. Cases were then classified according to the 2020 ECMM/ISHAM consensus criteria [4]. The study protocol was approved by the Medical University of

\footnotetext{
*Correspondence: joost.wauters@uzleuven.be; hoeniglmartin@gmail. com

${ }^{1}$ Division of Infectious Diseases, Excellence Center for Medical Mycology (ECMM), Medical University of Graz, Graz, Austria

${ }^{2}$ Medical Intensive Care Unit, Excellence Center for Medical Mycology

(ECMM), University Hospitals Leuven, Leuven, Belgium

Full author information is available at the end of the article
}

Graz (EC \#32-296 ex 19/20) and the other participating centers.

A total of 592 cases were entered (98.5\% from Europe; for demographics see Supplementary Table 1) of whom 11 (1.9\%) had proven CAPA, 80 (13.5\%) probable CAPA and $18(3 \%)$ possible CAPA. Table 1 displays diagnostic characteristics of those with CAPA. Median BALF-GM optical density index (ODI) in 83 CAPA patients was 2.74 (IQR 1.70-5.78). BALF-GM ODI $>1.0$ was also reported in $4 / 170(2.4 \%)$ patients without CAPA. BALF culture growing Aspergillus was detected exclusively among CAPA patients (0/184 non-CAPA patients). Serum GM was positive (>0.5 ODI) in $1 / 192$ patients without CAPA $(0.5 \%)$. Antifungal treatment details are depicted in Table 1 . Among those receiving systemic antifungal treatment, $52 \%$ were alive at ICU discharge.

During a median observation time of 32 days, 261 ICU deaths were observed. This corresponded to an -30 , -60 and -90 day ICU mortality of $36 \%$ (95\% CI 33-40), $46 \%$ (95\% CI 42-51) and 49\% (95\% CI 44-53). By applying a Fine-Gray competing risk model the 109 diagnosed CAPA cases (i.e., possible, probable or proven) on ICU corresponded to an $-1,-15$ - and -90 day ICU CAPA rate of $1 \%$ (95\% CI 1-3), $16 \%$ (95\% CI 13-20) and 20\% (95\% CI 16-32) (Supplementary Fig. 1).

Overall CAPA prevalence in our cohort was $15.4 \%$ and, therefore, within the range that has been previously reported in the literature [5], although prevalence varied widely between centers. Several factors such as awareness, diagnostic algorithms, local epidemiology and socioeconomic factors may explain these differences. 
Table 1 Diagnostic and treatment characteristics among CAPA patients

\begin{tabular}{|c|c|}
\hline & $\begin{array}{l}\text { Positivity in cases with possible/ } \\
\text { probable/proven CAPA }(n=109)^{*}\end{array}$ \\
\hline \multicolumn{2}{|l|}{ Diagnosis } \\
\hline BALF galactomannan $>1.0 \mathrm{ODI}$ & $64 / 83(77 \%)$ \\
\hline Serum galactomannan >0.5 ODI & $16 / 85(19 \%)$ \\
\hline Tracheal aspirate galactomannan $>1.2 \mathrm{ODI}$ & $16 / 21(76 \%)$ \\
\hline BALF-positive Aspergillus spp. culture & $45 / 85(53 \%)$ \\
\hline Bronchial aspiration positive Aspergillus spp. culture & $14 / 32(44 \%)$ \\
\hline Tracheal aspiration positive Aspergillus spp. culture & $42 / 68(62 \%)$ \\
\hline Sputum-positive Aspergillus spp. culture & $4 / 29(14 \%)$ \\
\hline BALF-positive Aspergillus PCR & $24 / 33(73 \%)$ \\
\hline Tracheal aspiration positive Aspergillus PCR & $7 / 32(22 \%)$ \\
\hline \multicolumn{2}{|l|}{ Treatment } \\
\hline Systemic antifungal treatment initiated for CAPA & $99 / 109(91 \%)$ \\
\hline Voriconazole & $52 / 99(53 \%)$ \\
\hline Isavuconazole & $36 / 99(36 \%)$ \\
\hline Lipid formulations of amphotericin B & $17 / 99(17 \%)$ \\
\hline Echinocandins & $13 / 99(13 \%)$ \\
\hline Deoxycholate amphotericin B & $3 / 99(3 \%)$ \\
\hline Posaconazole & $4 / 99(4 \%)$ \\
\hline $\begin{array}{l}\text { Antifungal combination therapy (voriconazole or isavuconazole based with echinocandin or liposomal ampho- } \\
\text { tericin B) }\end{array}$ & 18/99 (18\%) \\
\hline
\end{tabular}

BALF bronchoalveolar lavage fluid, CAPA COVID-19-associated pulmonary aspergillosis, ODI optical density index, $P C R$ polymerase chain reaction

*CAPA cases: Leuven, Belgium ( $n=9)$; Genoa, Italy $(n=5)$; Graz, Austria (2 centers, $n=13)$; Cologne, Germany $(n=19)$; Manchester, UK ( $n=2)$; Antwerp, Belgium $(n=12)$; Bruges, Belgium ( $n=2)$; Roeselare, Belgium $(n=1)$; Munich, Germany $(n=13)$; Madrid, Spain $(n=12)$; Nuernberg, Germany $(n=5) ;$ Stuttgart, Germany $(n=2)$; Bordeaux, France $(n=5)$; Karachi, Pakistan $(n=4)$; Ann Arbor, USA $(n=3)$; Besancon, France $(n=1)$; Modena, Italy $(n=1)$

Our results confirm that diagnosis of CAPA remains a challenge with limited sensitivity of serum GM (19\%) for CAPA and the need for a lower respiratory sample or tracheal/bronchial/lung biopsy to confirm diagnosis. Early diagnosis is considered a cornerstone in successful CAPA management, as ICU mortality rate is high in CAPA patients (52\% versus $39 \%$ in non-CAPA COVID-19 patients on ICU; $p=0.027$ ). Voriconazole and isavuconazole were most frequently used for antifungal monotherapy, following current treatment recommendations for CAPA [4]. Even though $>90 \%$ of CAPA patients received antifungal treatment, there was also one CAPA case not receiving antifungal therapy among the survivors, indicating that not all patients who are diagnosed with CAPA based on consensus definitions may in fact have invasive fungal disease.

\section{Supplementary Information}

The online version contains supplementary material available at https://doi org/10.1007/s00134-021-06471-6.

\section{Author details}

1 Division of Infectious Diseases, Excellence Center for Medical Mycology (ECMM), Medical University of Graz, Graz, Austria. ${ }^{2}$ Medical Intensive Care
Unit, Excellence Center for Medical Mycology (ECMM), University Hospitals Leuven, Leuven, Belgium. ${ }^{3}$ San Martino Polyclinic Hospital IRCCS, Genoa, Italy. ${ }^{4}$ Department of Health Sciences, University of Genoa, Genoa, Italy. ${ }^{5}$ Department of Microbiology, Immunology and Transplantation, KU Leuven, Leuven, Belgium. ${ }^{6}$ Department of Laboratory Medicine and National Reference Center for Mycosis, Excellence Center for Medical Mycology (ECMM), University Hospitals Leuven, Leuven, Belgium. ${ }^{7}$ Division of Infectious Diseases and Global Public Health, University of California San Diego, San Diego, CA, USA. ${ }^{8}$ Clinical and Translational Fungal-Working Group, University of California San Diego, San Diego, CA, USA.

\section{Acknowledgements}

We thank Marisa H. Miceli, Jean-Jacques Tudesq, Gregor Paul, Robert Krause, Marina Linhofer, Jonas Frost, Peter Zechner, Matthias Kochanek, Philipp Eller, Jeffrey D. Jenks, Sara Volpi, Anne-Pauline Bellanger, P Lewis White, Gustavo H. Goldman, Paul Bowyer, Antonis Rokas, Sara Gago, Paolo Pelosi, Chiara Robba, Jean-Pierre Gangneux, Cornelia Lass-Floerl, Marina Machado, and Patricia Muñoz. ECMM-CAPA Study Group Authors: Philipp Koehler, Jon Salmanton-García, Riina Rautemaa-Richardson, Stefan Hatzl, Johan Maertens, Yves Debaveye, Marc Bourgeois, Marijke Reynders, Lynn Rutsaert, Niels Van Regenmortel, Piet Lormans, Simon Feys, Alexander Christian Reisinger, Oliver A. Cornely, Tobias Lahmer, Maricela Valerio, Laurence Delhaes, Kauser Jabeen, Joerg Steinmann, Mathilde Chamula, Matteo Bassetti.

\section{Author contributions}

Substantial contribution to study concept and design: JP, JW, DRG, KL, MH. Substantial contribution to the acquisition of data for the work: JP, JW, DRG, JS-G, MB, MR, LR, NvR, PL, YD, JM, SF, ACR, TL, MV, LD, KJ, JS, SH, AR, MC, MH Substantial contribution to the statistical analysis or interpretation of data: JP, JW, DRG, SH, KL, MH. Drafting the manuscript: JP, MH. Critical review of the manuscript and final approval for publication: all authors. 


\section{Funding}

$\mathrm{MH}$ is supported by $\mathrm{NIH}$ and investigator initiated grants from Astellas, Gilead and Pfizer. No specific funding obtained for this study.

\section{Declarations}

\section{Conflicts of interest}

JP has received personal fees from Gilead Sciences and Pfizer, research funding from MSD and is stoke holder of AbbVie Inc and Novo Nordisk. JW reports grants and personal fees from Gilead and Pfizer: investigator-initiated grants, personal fees and also on-financial support from MSD. DRG reports an unconditional grant from Correvio Italia and a grant for his institution by Pfizer Inc. $\mathrm{KL}$ received consultancy fees from SMB Laboratories Brussels, MSD and Gilead, travel support from Pfizer, speaker fees from FUJIFILM WAKO, Pfizer and Gilead and a service fee from Thermo fisher Scientific. MH received research funding from Gilead Sciences, Astellas, Scynexis, F2G and Pfizer.

\section{Ethics approval}

The study protocol and all study-related procedures were approved by the Medical University of Graz (EC \#32-296 ex 19/20) and the other participating centers which all followed the local ethical requirements.

\section{Publisher's Note}

Springer Nature remains neutral with regard to jurisdictional claims in published maps and institutional affiliations.

Received: 18 June 2021 Accepted: 30 June 2021

Published online: 16 July 2021

\section{References}

1. Arastehfar A, Carvalho A, van de Veerdonk FL, Jenks JD, Koehler P, Krause R, Cornely OA, Perlin SD, Lass-Flörl C, Hoenigl M (2020) COVID-19 associated pulmonary aspergillosis (CAPA) — from immunology to treatment. J Fungi 6(2):91

2. Verweij PE, Rijnders BJA, Brüggemann RJM, Azoulay E, Bassetti M, Blot S, Calandra T, Clancy CJ, Cornely OA, Chiller T, Depuydt P, Giacobbe DR, Janssen NAF, Kullberg B-J, Lagrou K, Lass-Flörl C, Lewis RE, Liu PW-L, Lortholary O, Maertens J, Martin-Loeches I, Nguyen MH, Patterson TF, Rogers TR, Schouten JA, Spriet I, Vanderbeke L, Wauters J, van de Veerdonk FL (2020) Review of influenza-associated pulmonary aspergillosis in ICU patients and proposal for a case definition: an expert opinion. Intensive Care Med 46:1524-1535

3. Salmanton-García J, Sprute R, Stemler J, Bartoletti M, Dupont D, Valerio M, Garcia-Vidal C, Falces-Romero I, Machado M, de la Villa S, Schroeder M, Hoyo I, Hanses F, Ferreira-Paim K, Giacobbe DR, Meis JF, Gangneux JP, Rodríguez-Guardado A, Antinori S, Sal E, Malaj X, Seidel D, Cornely OA, Koehler P (2021) COVID-19-associated pulmonary aspergillosis, MarchAugust 2020. Emerg Infect Dis 27:1077-1086

4. Koehler P, Bassetti M, Chakrabarti A, Chen SCA, Colombo AL, Hoenigl M, Klimko N, Lass-Florl C, Oladele RO, Vinh DC, Zhu LP, Boll B, Bruggemann R, Gangneux JP, Perfect JR, Patterson TF, PersigehI T, Meis JF, OstroskyZeichner L, White PL, Verweij PE, Cornely OA, European Confederation of Medical M, International Society for Human Animal M, Asia Fungal Working G, Group ILIW, Group IPAMW, European Society for Clinical M, Infectious Diseases Fungal Infection Study G, Patients ESGfliCl, Interregional Association of Clinical M, Antimicrobial C, Medical Mycology Society of $\mathrm{N}$, Medical Mycology Society of China Medicine Education A, Infectious Diseases Working Party of the German Society for H, Medical O, Association of Medical M, Infectious Disease C, (2020) Defining and managing COVID-19-associated pulmonary aspergillosis: the 2020 ECMM/ISHAM consensus criteria for research and clinical guidance. Lancet Infect Dis. 21(6), e149-e162

5. Hoenigl M (2020) Invasive fungal disease complicating COVID-19: when it rains it pours. Clin Infect Dis Off Publ Infect Dis Soc Am. https://doi.org/ 10.1093/cid/ciaa1342 